\title{
Multi-wavelength View of the Interstellar Medium in the Large Magellanic Cloud
}

\author{
You-Hua Chu \\ Department of Astronomy, University of Illinois, 1002 W. Green Street, \\ Urbana, IL 61801, USA \\ chu@astro.uiuc.edu \\ Received 1997 August 1, accepted 1997 November 7
}

\begin{abstract}
The Large Magellanic Cloud (LMC) has been surveyed in optical emission lines, X-rays, radio continuum, $\mathrm{HI}$, and $\mathrm{CO}$ lines. These surveys provide views of the interstellar medium (ISM) in the LMC of unprecedented clarity, allowing us to study astrophysical processes and to examine the relationship among the different phases of the ISM. Multi-wavelength images are used to illustrate the physical structures of supernova remnants, superbubbles, and supergiant shells, as well as the global interstellar structure of the LMC.
\end{abstract}

Keywords: Magellanic Clouds — galaxies: ISM - ISM: general — ISM: structure — ISM: bubbles - supernova remnants

\section{Introduction}

The Large Magellanic Cloud (LMC) provides an ideal site for observational studies of the interstellar medium (ISM), as its small inclination $\left(30-40^{\circ}\right)$ minimises the confusion along the line of sight, its small distance $(50 \mathrm{kpc})$ affords high spatial resolutions, and its small foreground and internal extinctions (typically $A_{\mathrm{v}}<0.5 \mathrm{mag}$ ) offer unobscured views. Interstellar structures of the LMC can be studied in detail across the entire galaxy.

The ISM exists in multiple phases, including ionised gas at temperatures ranging from $10^{4} \mathrm{~K}$ to several $10^{6} \mathrm{~K}$, neutral atomic gas, and molecular gas. To achieve a comprehensive understanding of the physical structure and evolution of the ISM, one must study all of these components. Interstellar gas in different physical conditions emits preferentially in different wavelength ranges, while the sophistication of observing facilities for these wavelengths has been advancing disparately. It was not until recently that high-resolution radio and X-ray images of the LMC became available. We are finally able to examine the multiple-phase ISM of the LMC at multiple wavelengths in great detail.

\section{Multi-wavelength Observations of the Multi-phase ISM}

A variety of observing facilities has been used to study the multi-phase ISM of the LMC. For the $10^{4}$ $\mathrm{K}$ ionised gas, emission-line images were taken with a CCD camera on the Curtis Schmidt telescope at the Cerro Tololo Inter-American Observatory (CTIO), and long-slit high-dispersion spectra were taken with the echelle spectrograph on the CTIO $4 \mathrm{~m}$ telescope. The emission-line ( $\mathrm{H} \alpha$, [ÖII], and [SII]) images show the distribution and excitation of the ionised gas (Smith et al. 1998, present issue p. 163), while the echelle spectra show the internal motion and shocks in the ionised gas (e.g. Chu \& Kennicutt 1994).

The $10^{5} \mathrm{~K}$ ionised gas has been studied with interstellar absorption line observations in the UV, using Civ $\lambda \lambda 1548,1550$, Sirv $\lambda \lambda 1393,1402$ and Nv $\lambda \lambda 1238,1242$ lines. A large number of high-dispersion spectra of early-type stars in the LMC is available in the International Ultraviolet Explorer archives. These spectra are useful in diagnosing shocks inside superbubbles or supergiant shells, but provide no information on the hot gas halo of the LMC (Chu et al. 1994). The Goddard High Resolution Spectrograph (GHRS) on-board the Hubble Space Telescope (HST) has been used to take high-dispersion spectra with high $\mathrm{S} / \mathrm{N}$. With a careful selection of cool probe stars in quiescent interstellar environments, it is possible to determine not only the column density but also the kinematics of the $10^{5} \mathrm{~K}$ gas halo of the LMC (Wakker et al. 1998).

The $10^{6} \mathrm{~K}$ ionised gas is best observed in soft X-rays. The ROSAT X-ray satellite provides two detectors: the Position Sensitive Proportional Counter (PSPC) and the High Resolution Imager (HRI). The PSPC, sensitive to $0 \cdot 1-2 \cdot 4 \mathrm{keV}$, has an on-axis angular resolution of $\sim 30^{\prime \prime}$ and a spectral resolution of $45 \%$ at $1 \mathrm{keV}$. The HRI, sensitive to $0 \cdot 1-2 \cdot 0 \mathrm{keV}$, has an on-axis resolution of $\sim 5^{\prime \prime}$, but has essentially no spectral resolution. Both the PSPC and the HRI have been used to survey the 

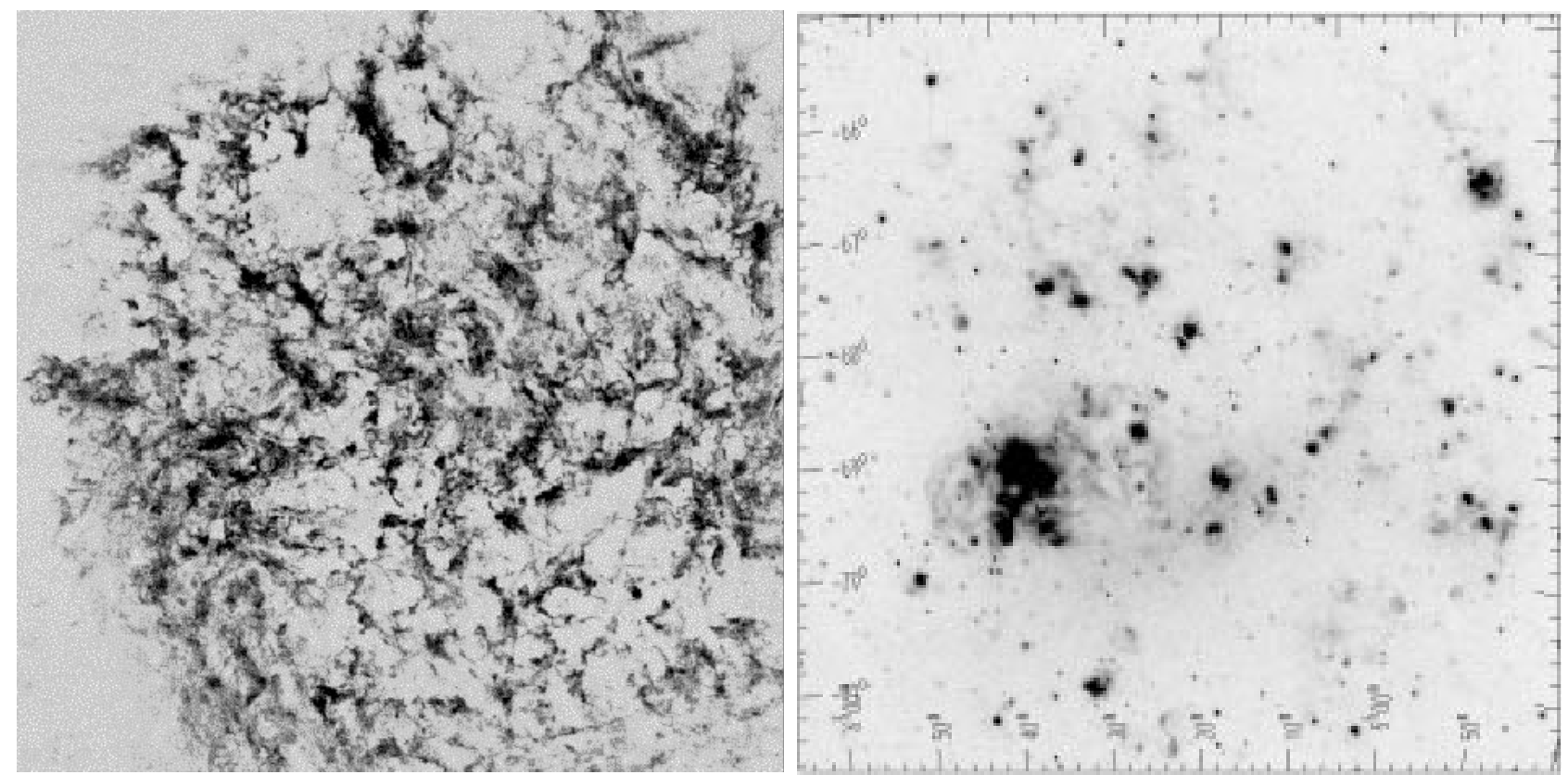

Figure 1- $\mathrm{HI}$ (left) and $\mathrm{H} \alpha$ (right) images of the LMC.

LMC (Snowden \& Petre 1994; Chu, Snowden \& Chang 1996).

The Hi distribution in the LMC has been mapped with the Parkes $64 \mathrm{~m}$ telescope at an angular resolution of $\sim 15^{\prime}$ (Rohlfs et al. 1984). An Hi mosaic of the LMC has recently been obtained with the Australia Telescope Compact Array (ATCA) at a resolution of $1^{\prime}$ (Kim et al. 1997a).

The molecular gas in the LMC has been mapped at a resolution of $8^{\prime} \cdot 8$ in the $J=1 \rightarrow 0$ rotational transition of CO (Cohen et al. 1988). A more recent CO survey of the LMC was carried out with the Swedish-ESO Submillimeter Telescope (SEST) at a resolution of $45^{\prime \prime}$ (Israel et al. 1993). The SEST CO maps of two star-formation regions, 30 Dor and N11, have been presented by Caldwell \& Kutner (1996).

\section{Structures of the Interstellar Medium in the LMC}

The structure of the ISM is determined by the interplay between massive stars and the ISM: molecular clouds form massive stars; massive stars dissociate, ionise, and dynamically structure the ambient medium; the ionised medium eventually cools, recombines, contracts under gravity, and forms molecular clouds. This simplistic picture can qualitatively explain the dramatically different structures of the ionised and neutral interstellar components in the LMC.

The structure of the molecular ISM in the LMC can be seen in the low-resolution CO map presented by Cohen et al. (1988). The large molecular concentrations are usually the sites of star formation activities. The most intense star formation region, the 30 Dor complex, sits on the northern tip of the largest and most massive concentration of molecular gas. In high-resolution maps of star forming regions, such as N11 (Caldwell \& Kutner 1996), remnant molecular gas is present and associated with the densest compact HiI regions.

The structure of the neutral atomic ISM in the LMC is revealed in detail in the ATCA Hi mosaic (Kim et al. 1997a). The differences between this ATCA mosaic and the Parkes $64 \mathrm{~m}$ map (Rohlfs et al. 1984) are clearly caused by the difference in resolution; however, the absence of a prominent $\mathrm{HI}$ ridge to the south of 30 Dor in the ATCA map might be indicative of a 'missing short baseline' problem, which prohibits the detection of wide features. The ATCA image, reproduced in the left panel of Figure 1, is dominated by $\mathrm{HI}$ filaments with interspersed holes across the entire galaxy. Hardly any correspondence between the Hi features and distributions of stars or other components of the ISM is obvious, besides the Hi cavities in the supergiant shells (SGSs) LMC 4 and LMC 5 (Kim et al. 1997a).

It is conceivable that the HI map should show a complex filamentary structure because it represents the cumulative effects of dynamic interactions of massive stars with the ISM in the past. If the HI maps are displayed with velocity resolution (in channel maps), correspondence between $\mathrm{HI}_{\mathrm{I}}$ holes and ionised gas shells may become clear (Kim et al. 1997b).

The structure of the $10^{4} \mathrm{~K}$ ionised interstellar gas is well depicted by $\mathrm{H} \alpha$ images, which can be obtained with high resolution $\left(\sim 1^{\prime \prime}\right)$ easily. The $\mathrm{H} \alpha$ image of the LMC displayed in the right panel of Figure 1 shows prominent HiI regions, SGSs with sizes up to $\sim 1400 \mathrm{pc}$, and diffuse gas that contributes up to $30-40 \%$ of the total $\mathrm{H} \alpha$ emission of the LMC 

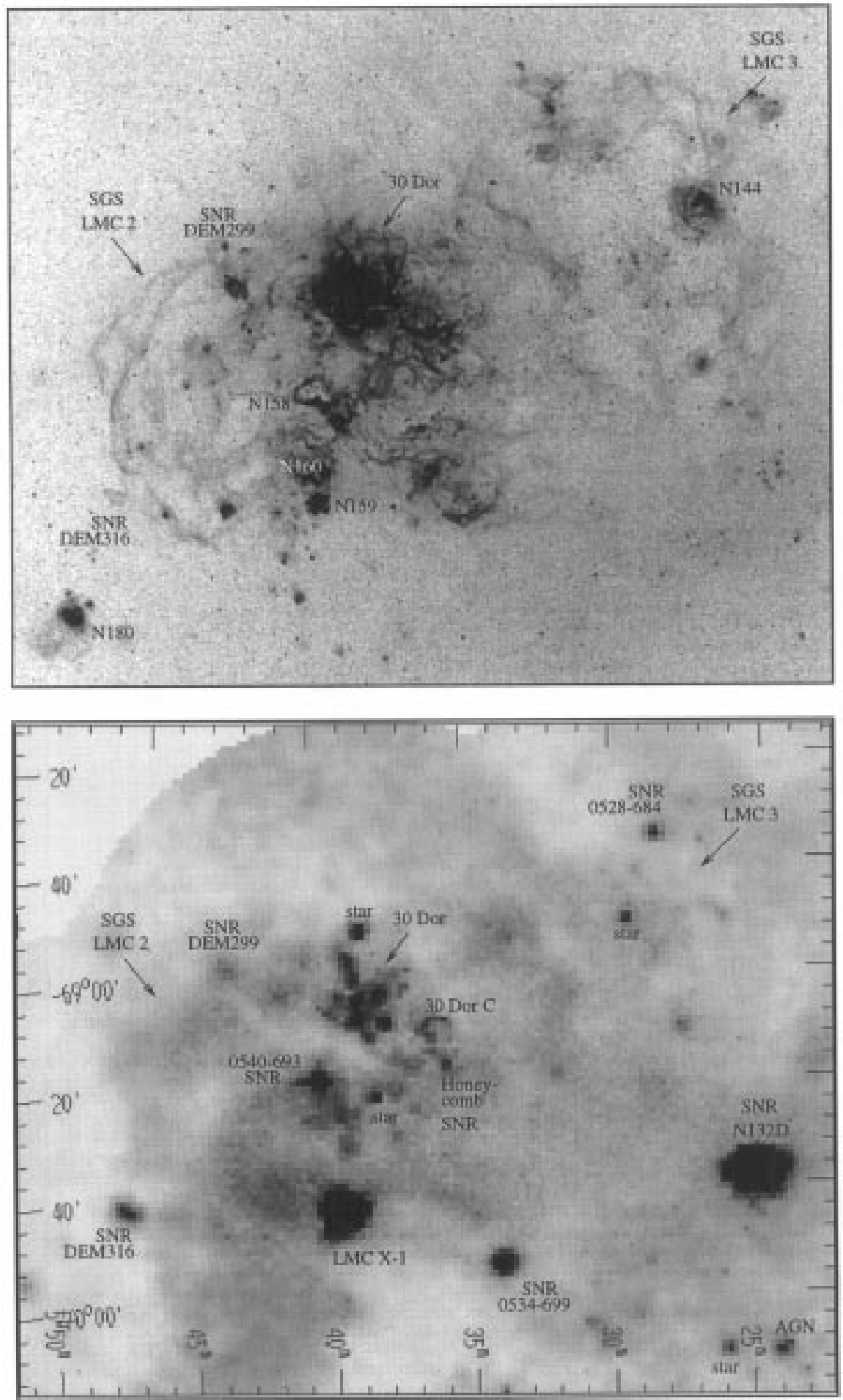

Figure 2- $\mathrm{H} \alpha$ (top) and X-ray (bottom) images of a portion of the LMC centred near the 30 Dor complex. Bright HiI regions, supernova remnants (SNRs), and supergiant shells (SGSs) are labeled. The Hir region and SNR names are from Henize (1956, "N'), Davies, Elliott, \& Meaburn (1976, 'DEM'), and Mathewson et al. (1983). 

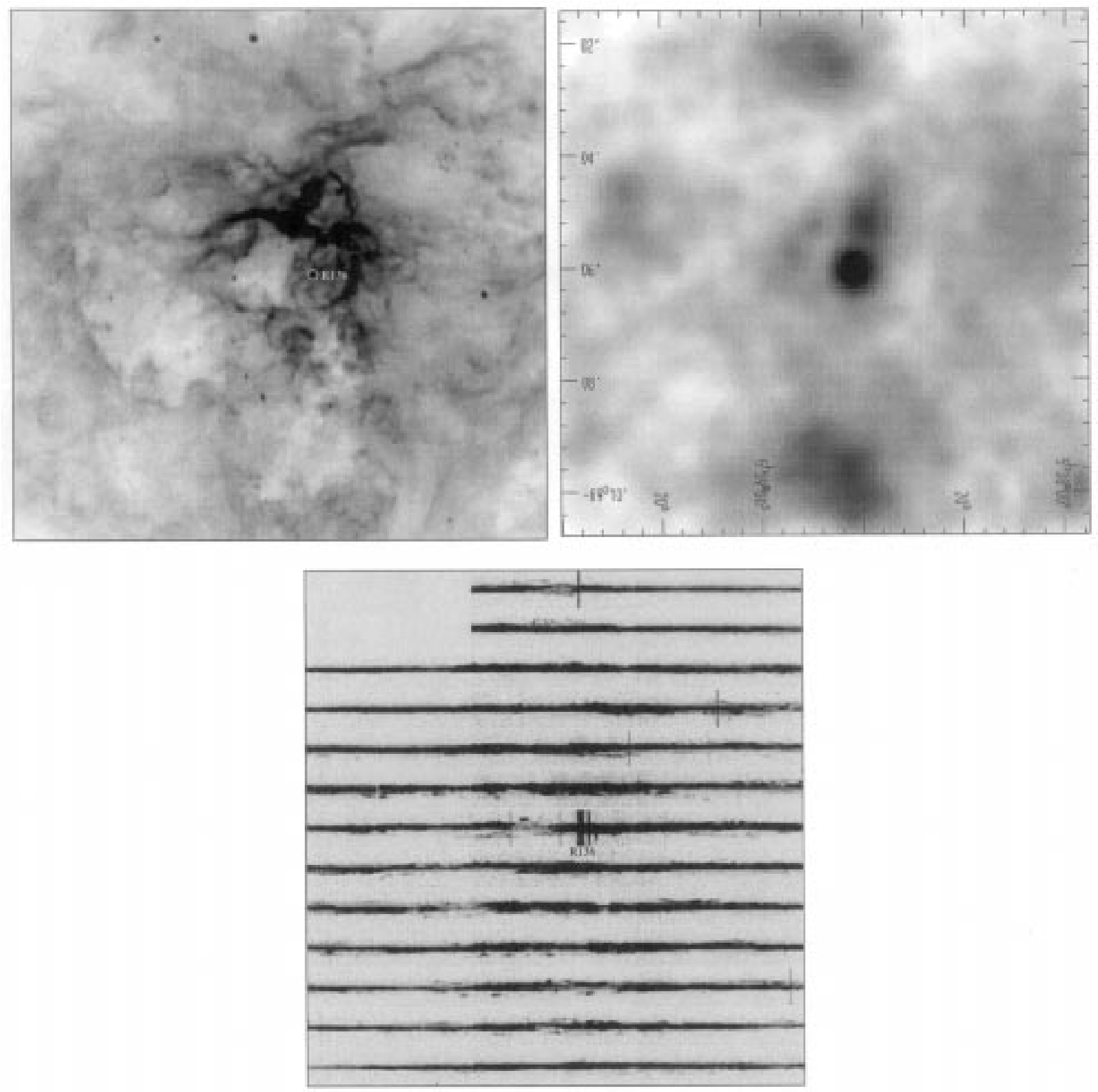

Figure 3- $\mathrm{H} \alpha$ (top left) and X-ray (top right) images of the central $9^{\prime} \times 9^{\prime}$ of 30 Doradus. The X-ray image is extracted from a ROSAT PSPC observation in the $0 \cdot 1-2 \cdot 4 \mathrm{keV}$ band. The bottom panel contains long-slit $\mathrm{H} \alpha$ echellograms of the same region, reproduced from Chu \& Kennicutt (1994). The wavelength increases upwards. The orientation is the same as the images above. The separation between adjacent slits corresponds to $45^{\prime \prime}$ in the sky or $600 \mathrm{~km} \mathrm{~s}^{-1}$ in velocity.

(Kennicutt et al. 1995). The $10^{4} \mathrm{~K}$ ionised gas is closely associated with massive stars. Massive stars are responsible for not only the ionisation of the medium, but also the dynamic structuring of the medium via fast stellar winds and supernova ejecta. Massive stars have short lifetimes, hence the structure of the $10^{4} \mathrm{~K}$ ionised ISM is freshly minted within the last $10^{7} \mathrm{yr}$. It is thus not surprising that the $\mathrm{H} \alpha$ and the HI images of the LMC (Figure 1) look so different.

Examined closely, the $10^{4} \mathrm{~K}$ ionised gas often show shell structures in supernova remnants (SNRs) and shell His regions around OB associations (superbubbles). The top panel of Figure 2 shows an $\mathrm{H} \alpha$ image of a portion of the LMC centred near the 30 Dor complex; the SGSs LMC 2 and LMC 3, the superbubbles in the HiI regions N158 and N160, and the large shells of SNRs DEM299 and DEM316 are clearly visible.

The $10^{6} \mathrm{~K}$ ionised gas is produced dynamically by fast stellar winds from massive stars or supernova ejecta. It is thus expected that some correspondence between the $10^{6} \mathrm{~K}$ ionised gas and the $10^{4} \mathrm{~K}$ ionised gas exists. Indeed, Figure 2 shows that the $10^{6}$ 
$\mathrm{K}$ ionised gas, as traced by soft X-ray emission, is detected in the interiors of SGSs, superbubbles, and SNRs, which have been identified by their His shells. The X-ray emission from the SGS LMC 4 has been analysed by Bomans et al. (1994); X-ray emission mechanisms for superbubbles have been summarised by Chu \& Mac Low (1996); and a multi-wavelength study of the SNR DEM316 has been reported by Williams et al. (1997).

The relationship between the $10^{4} \mathrm{~K}$ ionised gas and the $10^{6} \mathrm{~K}$ ionised gas and the production of the $10^{6} \mathrm{~K}$ gas by shocks are demonstrated in Figure 3, the central $9^{\prime} \times 9^{\prime}$ of 30 Doradus. It is evident that the bright diffuse X-ray emission is closely correlated with the presence of high-velocity, shocked material detected in the $\mathrm{H} \alpha$ echellograms.

On large scales, the distribution of the $10^{6} \mathrm{~K}$ gas shows features whose origin is not very clear. For example, the LMC bar contains only a few scattered His regions, but it displays bright diffuse $\mathrm{X}$-ray emission. Another example is the bright $\mathrm{X}$-ray spur extending to the south of the supergiant shell LMC 2; no optical counterpart is known or detected. The on-going ROSAT HRI survey of the LMC will provide high-resolution X-ray images and may help clarify the origin of this hot gas.

Finally, a $10^{5} \mathrm{~K}$ gas halo of the LMC has been unambiguously detected by Wakker et al. (1998) using HST GHRS observations of five probe stars. They find the hot gas halo to be patchy and expanding outward relative to the warm ionised gas (seen in $\mathrm{H} \alpha$ ) in the disk. Observations along more sightlines are needed to confirm these results and to determine the relationship between the $10^{5} \mathrm{~K}$ halo gas and the $10^{6} \mathrm{~K}$ hot gas readily visible in soft X-ray images.

\section{Acknowledgments}

YHC would like to thank R. C. Smith and R. C. Kennicutt for providing the $\mathrm{H} \alpha$ images, S. Kim and
L. Staveley-Smith for the Hi 21-cm mosaic, and S. L. Snowden for the ROSAT PSPC and HRI mosaics of the LMC. YHC acknowledges the support of NASA grants NAG 5-1900, NAG 5-3246, STI5787·01-94A, STI5894-02-94A, and STI6122·01-94A.

Bomans, D. J., Dennerl, K., \& Kürster, M. 1994, A\&A, 283, L21

Caldwell, D. A., \& Kutner, M. L. 1996, ApJ, 472, 611

Chu, Y.-H. 1995, Rev. Mex. AA (Conference Series), 3, 153

Chu, Y.-H., \& Kennicutt, R. C. 1994, ApJ, 425, 720

Chu, Y.-H. \& Mac Low, M.-M. 1996, in Röntgenstrahlung from the Universe, ed. H. U. Zimmermann, J. E. Trümper \& H. Yorke (Garching: MPE), p. 241

Chu, Y.-H., Snowden, S. L., \& Chang, T. 1996, The ROSAT Newsletter, No. 13, p. 26

Chu, Y.-H., Wakker, B., Mac Low, M.-M., \& García-Segura, G. 1994, AJ, 108, 1696

Cohen, R. S., Dame, T. M., Garay, G., et al. 1988, ApJ, 331, L95

Davies, R. D., Elliott, K. H., \& Meaburn, J. 1976, Mem. R. Astron. Soc., 81, 89

Henize, K. G. 1956, ApJS, 2, 315

Israel, F. P., et al. 1993, A\&A, 276, 25

Kennicutt, R. C., Bresolin, F., Bomans, D. J., Bothun, G. D., \& Thompson, I. B. 1995, AJ, 109, 594

Kim, S., Staveley-Smith, L., Sault, R. J., Kesteven, M. J., McConnell, D., \& Freeman, K. C. 1997a, PASA, 14, 119

Kim, S., Chu, Y.-H., Staveley-Smith, L., \& Smith, R. C. 1997b, ApJ (submitted)

Mathewson, D. S., Ford, V. L., Dopita, M. A., et al. 1983, ApJS, 51, 345

Rohlfs, K., Kreitschmann, J., Siegman, B. C., \& Feitzinger, J. V. 1984, A\&A, 137, 343

Smith, R. C., et al. 1998, PASA. 15, 163

Snowden, S. L., \& Petre, R. 1994, ApJ, 436, L123

Wakker, B., Howk, J. C., Chu, Y.-H., Bomans, D., \& Points, S. 1998, ApJ (submitted)

Williams, R. M., Chu, Y.-H., Dickel, J. R., Beyer, R., Petre, R., Smith, R. C., \& Milne, D. K. 1997, ApJ, 480, 618 